\title{
PICOT: A Trx- and Grx-Like Protein in Search of a Function
}

\author{
Ariel Ohayon, Guangyu Dong, Sagit Cohen, Moran Galperin, Alice Givoni, Keren Moyal and \\ Noah Isakov*
}

\begin{abstract}
The Shraga Segal Department of Microbiology and Immunology, Faculty of Health Sciences and the Cancer Research Center, Ben Gurion University of the Negev, P.O.B. 653, Beer Sheva 84105, Israel
\end{abstract}

\begin{abstract}
The PKC-interacting cousin of thioredoxin (PICOT) protein was discovered based on its ability to bind PKC $\theta$ in human T lymphocytes. Overexpression of PICOT was found to impose negative regulatory effects on PKC $\theta$-dependent functions. This included the inhibition of PKC $\theta$-dependent activation of c-Jun Nterminal kinase (JNK) and the activator protein 1 (AP-1) and nuclear factor kappa B (NF- $\mathrm{B}$ ) transcription factors. PICOT is a modular protein consisting of a single thioredoxin (Trx)-like homology domain (HD) and two highly homologous PICOT-HDs. The overall structure of each of the three domains resembles the canonical thioredoxin fold, which is common in enzymes that catalyze disulfide bond formation. Nevertheless, the three PICOT domains lack essential catalytic cysteine residues and their mode of activity is therefore unclear. PICOT is involved in the regulation of heart muscle function. Its overexpression in the heart of transgenic mice increased the ventricular function and cardiomyocyte contractility, and inhibited the overall cardiac hypertrophy induced by pressure overload. The effects of PICOT on the cardiac tissue are likely to be mediated via the muscle LIM protein (MLP), which was shown to interact with PICOT in cardiomyocytes, and colocalize with PICOT at the Z-disc of the sarcomer. PICOT interaction with MLP interfered with binding of the latter protein to the $\mathrm{Ca}^{2+}$-dependent Ser/Thr phosphatase, calcineurin, causing the displacement of calcineurin from the Z-disc. As a result, PICOT inhibited the calcineurin-mediated dephosphorylation and nuclear translocation of nuclear factor of activated T cells (NF-AT), and the transcriptional activation of NFAT regulated genes. Whether the effects of PICOT are dependent on PKC, and whether it can mediate catalytic activity and/or operate as an adaptor protein are only few of the open questions related to the biological mechanism of action of PICOT.
\end{abstract}

\section{INTRODUCTION}

Studies aimed at the identification of protein kinase $\mathrm{C}$ $(\mathrm{PKC})$ theta $(\mathrm{PKC} \theta)$ regulatory molecules, have led Witte et al. to the discovery of a new gene product, termed PICOT (PKC-interacting cousin of thioredoxin), which was found to possess the ability to associate with $\mathrm{PKC} \theta$ in human $\mathrm{T}$ lymphocytes [1]. PICOT was first isolated using a yeast-two hybrid screen of human Jurkat T cell cDNA library with bait that consisted of a catalytically inactive full-length PKC $\theta$ cDNA.

The human PICOT predominates in the cell's cytoplasm; it has a molecular mass of $\sim 37 \mathrm{kDa}$ and includes 335 aa. Its N-terminal domain share sequence homology with members of the thioredoxin family, but is missing a conserved motif (Cys-Gly-Pro-Cys) essential for the thioredoxin catalytic activity. It mediates however binding to PKC $\theta$. The $\mathrm{C}$ terminal includes a tandem repeat of an evolutionary conserved domain, termed PICOT-HD, which is shared by proteins from diverse groups of organisms (Fig. 1) [2].

Transient overexpression of constitutively active PKC $\theta$ in Jurkat $\mathrm{T}$ cells resulted in upregulation of the c-Jun $\mathrm{N}$ -

*Address correspondence to this author at the Shraga Segal Department of Microbiology and Immunology, Faculty of Health Sciences, Ben Gurion University of the Negev, P.O.B. 653, Beer Sheva 84105, Israel;

E-mail: noah@bgu.ac.il terminal kinase (JNK) activity, an effect that was partially inhibited by overexpressed PICOT. In addition, PICOT inhibited JNK activation induced by cotransfected constitutively active PKC $\theta$ and calcineurin, which are known to cooperate in the activation of JNK in antigen stimulated T cells [3]. Further studies demonstrated that PICOT inhibits PKC $\theta$ dependent transcriptional activation mediated by the AP-1 and $\mathrm{NF}-\kappa \mathrm{B}$ transcription factors [1].

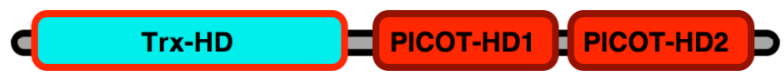

Fig. (1). A schematic structure of the human PICOT protein. The protein is 335 aa long and includes three conserved domains: an $\mathrm{N}$ terminal thioredoxin (Trx)-like homology domain (HD)(aa12-143), and two 84 aa long PICOT-HDs (aa 145-228 and 247-330) that exhibit $64 \%$ identity in sequence. PICOT is also termed 'thioredoxin-like 2' (TXNL2) by the HUGO gene nomenclature, and 'glutaredoxin 3' (Glrx3) by the Mouse Genome Informatics Database.

$\mathrm{T}$ cell staining with PICOT specific antibodies (Abs) demonstrated that the protein is expressed predominantly in the cytoplasm. In addition, our own studies revealed that overexpression of PICOT in different cell lines resulted in predominant localization of the transfected gene product in the cytoplasm, with relatively low levels (that differ from one cell line to another) found in the nucleus (see Fig. 2). Recent studies by Park and colleagues $[4,5]$ demonstrated 
that PICOT has a significant regulatory role in heart muscle function where it can attenuate stress-induced cardiac hypertrophy. The present review highlights some of the most recent findings on PICOT and discusses the potential biological function of this protein.
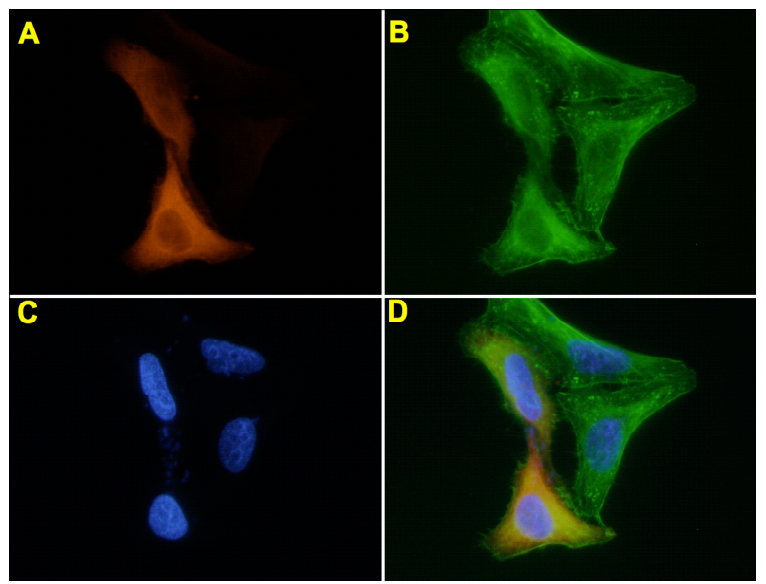

Fig. (2). Localization of overexpressed PICOT in HepG2 cells by immunofluorescence staining. HepG2 cells were transfected with pEF-PICOT, cultured for 3 days, fixed, and stained with rabbit antiPICOT Abs and a PE-conjugated anti-rabbit $\operatorname{IgG}(\mathbf{A})$, phalloidin that specifically binds filamentous actin (B), and DAPI, which associates with the minor groove of the double strand DNA (C). A merged image is shown in D. PICOT localize predominantly in the cell's cytoplasm, with low levels at the nucleus.

\section{STRUCTURE OF PICOT AND RELATIONSHIP TO THIOREDOXIN AND GLUTAREDOXIN}

The biological role of PICOT has not been identified yet, nor is the exact physiological function of its isolated Trx- or PICOT-HD. Many different proteins from a wide range of species were found to include one or more PICOT-HD as part of the molecule. In a large number of such proteins, the PICOT-HD was found at the protein's C-terminus, in close proximity to N-terminal Trx- or Grx-like domains [2]. The close physical proximity between PICOT-HD and Trx- or Grx-HD in multiple proteins suggests functional relationships between the three domains. Furthermore, PICOT, TrXHD, and Grx-HD possess an overall similar globular topology [6]. Each of the three domains possesses the canonical 'thioredoxin fold domain', formed by a central mix of 4 or 5 strand $\beta$-sheets flanked by three or more $\alpha$-helices on either side of the $\beta$-strands $[7,8]$ (see Fig. 3). The results imply that formation of the three domains during evolution originated from a single common ancestral gene. It should be mentioned however that several genes encode short proteins, which are built almost entirely of a PICOT-HD. It appears therefore that PICOT-HD-containing proteins may have biological functions that are independent of Trx or Grx, or that can operate in conjunction with other molecules, such as Trx or Grx.

\section{INVOLVEMENT OF PICOT IN SIGNAL TRANSDUC- TION}

The original discovery of PICOT was based on its ability to interact with $\mathrm{PKC} \theta$ [1], a critical enzyme for $\mathrm{T}$ cell antigen receptor (TCR)-linked signal transduction pathway $[10,11]$. PKC $\theta$ is a $\mathrm{Ca}^{2+}$-independent $\mathrm{PKC}$ isoform that plays an essential role in reorganization and/or function of the immunological synapse $[12,13]$. It cooperates with calcineurin in activation of the interleukin-2 (IL-2) gene [3], and is involved in the promotion of signaling pathways that regulate $\mathrm{T}$ cell activation and survival [14-16]. Initial studies demonstrated that PICOT can serve as a negative regulator of $\mathrm{PKC} \theta$ in $\mathrm{T}$ cells, since its overexpression resulted in downregulation of $\mathrm{PKC} \theta$-dependent activation of JNK, concomitantly with inhibition of the transcription factors AP-1 and NF-KB [1].

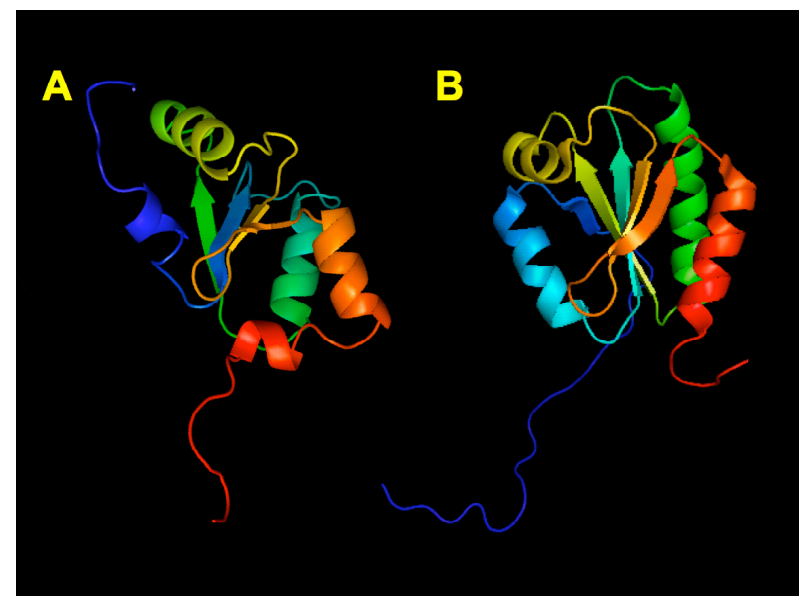

Fig (3). The 3D structure of the mouse PICOT-HD2 (A) and the human PICOT Trx-HD (B) were solved by K. Miyamoto et al. (PDB, 1WIK) and N. Tochio et al. (PDB, 2DIY), respectively. The figures were prepared using the PyMol Molecular Graphics System [9].

Physiological activation of T cells is initiated by TCR interaction with a major histocompatibility complex (MHC)bound peptide antigen on the surface of antigen presenting cells (APC). The very early phase of the TCR-linked signal transduction pathway is regulated by protein tyrosine kinases (PTKs), which phosphorylate the cytoplasmic tails of the TCR associated CD3 subunits, as well as a plethora of effector molecules that are involved in signal transduction and induction of the activation response. Many of the effects induced by a physiological activation of tyrosine kinases can be mimicked by cell treatment with reactive oxygen intermediates (ROS), such as hydrogen peroxide, that were recently found to be produced in vivo and serve as physiological regulators of lymphocyte activation [17]. Other cell types were also found to produce ROS in response to cytokines and growth factors $[18,19]$. One of the major sources for inducible ROS in leukocytes is the NADPH oxidase, which can be activated by several mechanisms, including phosphorylation by PKC [20].

The fact that PICOT associates with PKC $\theta$ and possesses Trx- and Grx-like sequences suggested that it might be involved in redox-regulated biochemical pathways. Treatment of Jurkat $\mathrm{T}$ cells with hydrogen peroxide resulted in phosphorylation of PICOT on tyrosine residues, an effect that could be abolished by inhibitors of Src family members of PTKs [21]. Furthermore, tyrosine phosphorylation of PICOT 
was also observed in untreated cells following their transfection with a constitutively active Lck (Lck Y505F). The results suggest that tyrosine phosphorylation of PICOT can be directly or indirectly mediated by Lck. They also imply that PICOT plays a role in cell-activation dependent signaling pathways, and/or responses to stress signals mediated by reactive oxygen intermediates.

The fact that PKC $\theta$ is expressed predominantly in hematopoietic and muscle cells, in contrast to PICOT, which is expressed in a wide range of tissues, suggests that PICOT is likely to be involved in biological functions that are independent of PKC $\theta$.

\section{THE ROLE OF PICOT IN THE REGULATION OF HEART MUSCLE FUNCTION}

The potential involvement of PICOT in cellular processes regulating heart muscle function was demonstrated by Jeong et al. during studies of the mechanisms leading to cardiac hypertrophy $[4,5]$. These studies were aimed at the identification of potential regulators of cardiac hypertrophy, and led to the observation that PICOT was among several genes undergoing upregulation following transverse aortic constriction-induced cardiac hypertrophy of adult rat hearts. Furthermore, PICOT overexpression in the heart of transgenic mice inhibited cardiac hypertrophy induced by pressure overload, concomitantly with increase in ventricular function and cardiomyocyte contractility.

Jeong et al. have further analyzed the potential binding partners of PICOT in the muscle tissue, and using a GSTPICOT pull-down assay in conjunction with mass spectrometry they found that PICOT can directly interact with the muscle LIM domain protein (MLP) [4, 5], a member of a family of cysteine-rich proteins that mediate protein-protein interactions [22]. MLP is known to interact with several different proteins, including $\alpha$-actinin, zyxin and calcineurin, all of which are known to concentrate at the Z-disc of the sarcomer [23, 24]. Immunofluorescence staining of tissue section of adult mouse heart using PICOT-specific Abs demonstrated PICOT localization within the Z-disc [5], results that were confirmed by our own studies (see Fig. 4). In addition, PICOT was found to co-localize with MLP and another known Z-disc protein, the $\alpha$-actinin [5]. MLP interaction with calcineurin, a $\mathrm{Ca}^{2+}$-dependent Ser/Thr phosphatase, is known to be essential for calcineurin anchorage to the Z-disk and upregulation of its catalytic activity. The active, calcineurin then dephosphorylates the cytoplasmic NFAT transcription factor and promotes its translocation to the nucleus where it induces the transcription of NF-AT regulated genes [23]. Overexpression of PICOT and its association with MLP interfered with MLP binding to calcineurin, causing displacement of calcineurin from its anchorage site at the $\mathrm{Z}$ disc. As a result, PICOT inhibited calcineurinmediated dephosphorylation and subsequent nuclear translocation of NF-AT, as well as the NF-AT-regulated gene transcription. The authors suggest that PICOT inhibits cardiac hypertrophy by negative regulation of the MLP-calcineurinNF-AT signaling pathway via a mechanism, which disrupts MLP-calcineurin interaction.

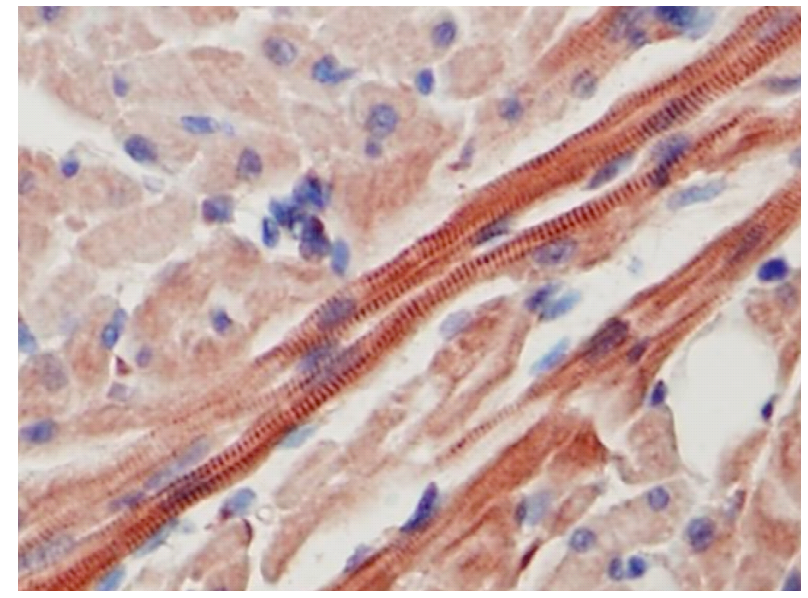

Fig. (4). Immunohistochemical staining of adult mouse heart sections with anti-PICOT Abs. Paraffin embedded sections of adult mouse heart were deparaffinized, mounted on glass slides, blocked with BSA, treated with hydrogen peroxide, and stained with rabbit anti-PICOT Abs plus biotinylated goat anti-rabbit IgG and peroxidase-conjugated streptavidin. Peroxidase activity was demonstrated by incubation with APC and hydrogen peroxide. Slides were counterstained with Hematoxylin and examined under a bright field microscope (Olympus I-70) attached to a digital camera (Olympus DP70).

\section{POTENTIAL ROLE FOR PICOT DURING EMBRYO- INC DEVELOPMENT}

Comparative analysis of protein profiles expressed at different stages of embryonic development serves as a useful tool for the identification of molecules that are involved in specific developmental processes. In order to determine protein profile changes during embryogenesis Greene et al. [25] have utilized an immobilized $\mathrm{pH}$ gradient-based twodimensional electrophoresis and identified individual protein spots by mass spectrometry. Analysis of proteins expressed between days (E) 8.5-to-10.5 of embryogenesis, the time of formation of the neural tube, revealed a number of changes in protein patterns at successive embryonic days. PICOT was almost undetectable at E8.5 but was dramatically upregulated at E9.5. Although the onset of PICOT expression correlated with that of PKCO [26], the incomplete overlap in tissue expression of the two proteins suggest that PICOT may have developmental roles that are independent of PKC $\theta$.

\section{CONCLUSIONS}

Despite the fact that the exact biological activity of PICOT is not yet known, indirect studies suggest that PICOT plays important roles in the regulation of $\mathrm{T}$ lymphocyte activation and during cardiac muscle responses to hypertrophyinducing signals. PICOT is also predicted to be involved in certain developmental stages in mouse embryos, although the exact role or the tissue in which it is first expressed are not know. PICOT was shown to associate with PKC $\theta$ in $\mathrm{T}$ cells, but is also expressed in PKC $\theta$-negative cell types indicating that PICOT assumes activities independent of PKC $\theta$. Further studies of this novel protein and characterization of its exact biological activity may reveal important clues about 
PICOT and the biological processes that are mediated by or dependent on PICOT.

\section{ACKNOWLEDGEMENTS}

Research in our laboratory is supported in part by the Israel Science Foundation, USA-Israel Binational Science Foundation, Israeli-Taiwanese Scientific Research Cooperation, Israel Cancer Research Fund, Chief Scientist's office, Israel Ministry of Health, the Israel Cancer Association through a donation be Ida and Harry Shooster, and a donation by Linda Osofsky. N.I. holds the Joseph H. Krupp Chair in Cancer Immunobiology.

\section{REFERENCES}

[1] Witte S, Villalba M, Bi K, Liu Y, Isakov N, Altman A. Inhibition of the c-Jun N-terminal kinase/AP-1 and NF-kappaB pathways by PICOT, a novel protein kinase $\mathrm{C}$-interacting protein with a thioredoxin homology domain. J Biol Chem 2000; 275(3): 1902-9.

[2] Isakov N, Witte S, Altman A. PICOT-HD: a highly conserved protein domain that is often associated with thioredoxin and glutaredoxin modules. Trends Biochem Sci 2000; 25(11): 537-9.

[3] Werlen G, Jacinto E, Xia Y, Karin M. Calcineurin preferentially synergizes with PKC-theta to activate JNK and IL-2 promoter in T lymphocytes. EMBO J 1998; 17(11): 3101-11.

[4] Jeong D, Cha H, Kim E, et al. PICOT inhibits cardiac hypertrophy and enhances ventricular function and cardiomyocyte contractility. Circ Res 2006; 99(3): 307-14.

[5] Jeong D, Kim JM, Cha H, et al. PICOT attenuates cardiac hypertrophy by disrupting calcineurin-NFAT signaling. Circ Res 2008; 102(6): 711-9.

[6] Herrero E, de la Torre-Ruiz MA. Monothiol glutaredoxins: a common domain for multiple functions. Cell Mol Life Sci 2007; 64 (12): 1518-30.

[7] Kinch LN, Baker D, Grishin NV. Deciphering a novel thioredoxinlike fold family. Proteins 2003; 52(3): 323-31.

[8] Qi Y, Grishin NV. Structural classification of thioredoxin-like fold proteins. Proteins 2005; 58(2): 376-88.

[9] Delano WL. The PyMOL Molecular Graphics System. DeLano Scientific; San Carlos, CA. 2002.

[10] Altman A, Isakov N, Baier G. Protein kinase Ctheta: a new essential superstar on the T-cell stage. Immunol Today 2000; 21(11): 567-73.

[11] Isakov N, Altman A. Protein kinase $\mathrm{C}$ (theta) in $\mathrm{T}$ cell activation. Annu Rev Immunol 2002; 20: 761-94.

[12] Bi K, Tanaka Y, Coudronniere N, et al. Antigen-induced translocation of PKC-theta to membrane rafts is required for T cell activation. Nat Immunol 2001; 2(6): 556-63.
[13] Arendt CW, Albrecht B, Soos TJ, Littman DR. Protein kinase Ctheta;: signaling from the center of the T-cell synapse. Curr Opin Immunol 2002; 14(3): 323-30.

[14] Bertolotto C, Maulon L, Filippa N, Baier G, Auberger P. Protein kinase $\mathrm{C}$ theta and epsilon promote $\mathrm{T}$-cell survival by a rskdependent phosphorylation and inactivation of BAD. J Biol Chem 2000; 275(47): 37246-50.

[15] Villalba M, Bushway P, Altman A. Protein kinase C-theta mediates a selective $\mathrm{T}$ cell survival signal via phosphorylation of BAD. J Immunol 2001; 166(10): 5955-63.

[16] Manicassamy S, Gupta S, Huang Z, Sun Z. Protein kinase C-thetamediated signals enhance $\mathrm{CD} 4+\mathrm{T}$ cell survival by up-regulating Bcl-xL. J Immunol 2006; 176(11): 6709-16.

[17] Reth M. Hydrogen peroxide as second messenger in lymphocyte activation. Nat Immunol 2002; 3(12): 1129-34.

[18] Meier B, Radeke HH, Selle S, et al. Human fibroblasts release reactive oxygen species in response to interleukin-1 or tumour necrosis factor-alpha. Biochem J 1989; 263(2): 539-45.

[19] Ohba M, Shibanuma M, Kuroki T, Nose K. Production of hydrogen peroxide by transforming growth factor-beta 1 and its involvement in induction of egr-1 in mouse osteoblastic cells. J Cell Biol 1994; 126(4): 1079-88

[20] Reeves EP, Dekker LV, Forbes LV, et al. Direct interaction between $\mathrm{p} 47$ phox and protein kinase $\mathrm{C}$ : evidence for targeting of protein kinase C by p47phox in neutrophils. Biochem J 1999; 344 (Pt 3): 859-66.

[21] Babichev $\mathrm{Y}$, Gelkop S, Witte S, Altman A, Isakov N. The protein kinase $\mathrm{C}$ (PKC) theta interacting protein, PICOT, undergoes tyrosine phosphorylation in response to hydrogen peroxide. In: 'Proceedings of the 2nd congress of the Federation of Immunological Societies of Asia-Oceania (FIMSA 2000)', Mundozzi Ed., 2000; 11-6.

[22] Bach I. The LIM domain: regulation by association. Mech Dev 2000; 91(1-2): 5-17.

[23] Heineke J, Ruetten H, Willenbockel C, et al. Attenuation of cardiac remodeling after myocardial infarction by muscle LIM proteincalcineurin signaling at the sarcomeric Z-disc. Proc Natl Acad Sci USA 2005 ; 102(5): 1655-60.

[24] Hoshijima M. Mechanical stress-strain sensors embedded in cardiac cytoskeleton: $\mathrm{Z}$ disk, titin, and associated structures. Am J Physiol Heart Circ Physiol 2006; 290(4): H1313-25.

[25] Greene ND, Leung KY, Wait R, Begum S, Dunn MJ, Copp AJ. Differential protein expression at the stage of neural tube closure in the mouse embryo. J Biol Chem 2002; 277(44): 41645-51.

[26] Wilda M, Ghaffari-Tabrizi N, Reisert I, Utermann G, Baier G, Hameister H. Protein kinase C isoenzyme: selective expression pattern of protein kinase C-\&theta; during mouse development. Mech Dev 2001; 103(1-2): 197-200.

(C) Ohayon et al.; Licensee Bentham Open.

This is an open access article distributed under the terms of the Creative Commons Attribution License (http://creativecommons.org/licenses/by/2.5/), which permits unrestrictive use, distribution, and reproduction in any medium, provided the original work is properly cited. 